\title{
Investigating the Time Course of Part-Based and Holistic Processing in Face Perception
}

\section{Chao-Chih Wang*}

Research Center for Education and Mind Sciences, Hsinchu Teachers College, National Tsing Hua University, Hsinchu, Taiwan

Human has an exceptional ability for face recognition to keep up social network. However, it is unclear to understand the mechanisms of face recognition until now. Specifically, there is less research to examine the time course of part-based and holistic processing when these two routes trigger and finish. In the present experiments, the exposure time was manipulated to examine the time course of face processing and found evidence suggesting that holistic processing occurs shortly after part-based processing at about $200 \mathrm{~ms}$, and can last for a relatively long duration up to 2,000 ms. These results may support to a dual-route model comprising holistic processing and

OPEN ACCESS

Edited by:

Satoshi Hirata

Kyoto University, Japan

Reviewed by:

Yoshiyuki Ueda,

Kyoto University, Japan Juha Silvanto,

University of Westminster, United Kingdom

*Correspondence: Chao-Chih Wang ccu.george@gmail.com

Specialty section: This article was submitted to

Perception Science, a section of the journal

Frontiers in Psychology

Received: 11 January 2018 Accepted: 06 December 2018 Published: 17 January 2019

Citation: Wang C-C (2019) Investigating the Time Course of Part-Based and Holistic Processing in Face

Perception. Front. Psychol. 9:2630. doi: 10.3389/fpsyg.2018.02630 part-based processing in face perception. Moreover, our findings were inconsistent with the previous study which suggests that no holistic processing was observed at the relatively long duration, and suspected that perceptual discriminability may have been responsible for the discrepancy.

Keywords: face perception, part-based processing, holistic processing, time course, composite face illusion, dual-route model

\section{INTRODUCTION}

Ever since Galton's (1879) insight that face recognition is most likely based on integration of facial features, holistic processing has been construed as a very important component to understand how humans perceive faces (Young et al., 1987; Tanaka and Gordon, 2011). Face perception is a distinct human ability for recognizing members of your family, friends and foes, celebrities and enemies, even caricatures and cartoons. The same level of ease and efficiency of face recognition performance is difficult to meet even with the enormous information-processing and computational power of advanced computers today, largely because the underlying processing seems so complicated that the proper level of algorithms for achieving the task is not clear for engineers (Richler et al., 2012). Although humans could recognize thousands of people, oftentimes flawlessly, many questions remain as to how this unusual ability of face recognition is achieved. In their seminal and influential article, Bruce and Young (1986) proposed the first functional model to explain how human recognizes familiar faces (see Figure 1). According to Bruce and Young, recognizing familiar faces entails a match between information of structural encoding of the face that is present and stored codes of face recognition units (FRUs). Subsequently, identity-specific semantic codes are processed and created from person identity nodes and name codes are retrieved. Cognitive system plays the role of deciding whether or not the initial match is adequately close for veridical 
recognition or only a degree of likeness, and takes into account a number of factors before reaching the final decision.

Bruce and Young's (1986) functional model has been credited for accounting for many aspects of (familiar) face processing in terms of the relevant codes. Although structural encoding may well comprise both part-based processing and configural processing, Bruce and Young did not explicitly make that distinction. On the other hand, Maurer et al. (2002) had proposed that there are three types of configural processing: detecting the presence of first-order spatial relations that define a face, holistic processing that integrates facial features into a gestalt whole, and processing of second-order spatial relations that is sensitive to spacing and metric information among facial features. McKone (2010), however, contended that there is no convincing evidence for dissociation or separation between holistic processing and processing of second-order relations. Instead, she argued that there are many theoretical reasons to consider holistic processing to be conducted on a single integrated representation, which she would dub as holistic/configural processing, rather than comprising a number of separate subcomponents (McKone, 2010). As yet another alternative, there could be a compromise between the view espoused by Maurer and her colleagues on one hand and that by McKone on the other. That is, the processing of face recognition system may be divided into two subcomponents, comprising face detection and holistic face processing, because there is convincing evidence to support the separation between these two parts (Maurer et al., 2002; McKone, 2010).

Moreover, McKone had proposed a dual-route model for face processing where holistic/configural face recognition system and part-based visual recognition system operate independent of each other concretely. According to McKone (2010, see also McKone and Yovel, 2009), holistic processing comprises extraction of a variety of information from a face, including spacing between centers of major nameable facial features such as interocular distance, nose-mouth distance, as well as overall position of features in a face, while excluding shape details of local features. As illustrated in Figure 2, McKone (2010) suggested that holistic/configural face recognition system diverges from part-based visual recognition system after the point where face processing has gone through mid-level visual processing. After that, the products of midlevel processing are fed into two parallel routes for further processing, representing, respectively, the part-based visual cognition system and the holistic/configural recognition system. Neuropsychological evidence from patient studies seems to support that there are dissociations between partbased visual recognition system and holistic/configural face recognition system. For example, patient $\mathrm{CK}$ suffered from object agnosia and dyslexia as a consequence of a closedhead injury, but was intact for face recognition (Moscovitch et al., 1997). In contrast, McNeil and Warrington (1993) reported that the patient WJ exhibited severe prosopagnosia (impaired ability in face recognition) but had intact ability in object recognition. Moreover, some studies indicated that right occipital face area and fusiform face area play an important role for holistic processing (Kanwisher et al., 1997;
McKone, 2010; Jonas et al., 2012, 2014; Bona et al., 2016).

What exactly are the differences between part-based visual recognition system and holistic/configural face recognition system? There is a main issue needs to be addressed concerns whether or not the part-based processing can trigger holistic processing such that albeit structurally separated their functional operations are related or whether they are strictly independent of each other with no possibility of functional links as McKone had proposed (see Figure 2). Thus far, the evidence has been inconsistent: For example, Amishav and Kimchi (2010) suggested that both componential and configural processing are integral for upright faces because participants could not selectively perceive features without simultaneously being influenced by configurational variation and vice versa. Their findings appear to implicate that holistic processing and part-based processing did not operate independently. On the other hand, Fitousi (2015, 2016) employed the system factorial technology (SFT) as a means for mathematical modeling of the nature of face processing, and argued that face recognition is achieved analytically rather than holistically. Specifically, he was able to demonstrate the existence of composite face effect, but failed to obtain Garner interference and super-capacity with the same composite faces. These results are consistent with analytic processing (Fitousi, 2015, 2016). Therefore, further studies are required to help the controversial issue.

There are at least three important questions need to be answered with regard to McKone's (2010) dual-route model: first, as discussed earlier, if holistic/configural face recognition system is unitary rather than comprising subcomponents, how would the dual-route model explain the fact that people who suffer from prosopagnosia still can detect the presence of a face, while fail to recognize familiar faces? If people afflicted with prosopagnosia can nevertheless detect the presence of a face, it would imply that there is dissociation between face detection and holistic processing. Second, is there evidence to support the hypothesis regarding the time course to demonstrate separation between part-based processing and holistic/configural processing? If the results suggest temporal differences in the operation of these two modes of processing, that would provide credence to support the separation between part-based visual recognition and holistic/configural face recognition systems. On the other hand, if the results do not suggest temporal differences in the operation of these two modes of processing, it does not necessarily implicate whether these two modes of processing are interactive or whether they can operate independently. In other words, logically speaking, the difference in time course is a sufficient condition but not necessary condition for arguing the separation between part-based and holistic/configural holistic processing. Moreover, the separation between two modes of processing does not necessarily predict differences in time course. For example, the part-based processing and holistic processing can operate in parallel and simultaneously with no temporal difference, as seems to be implied by McKone's model depicted in Figure 2. Finally, both the part-based visual recognition and holistic/configural face recognition systems are temporally subsequent to the mid-level vision. However, it is not clear 


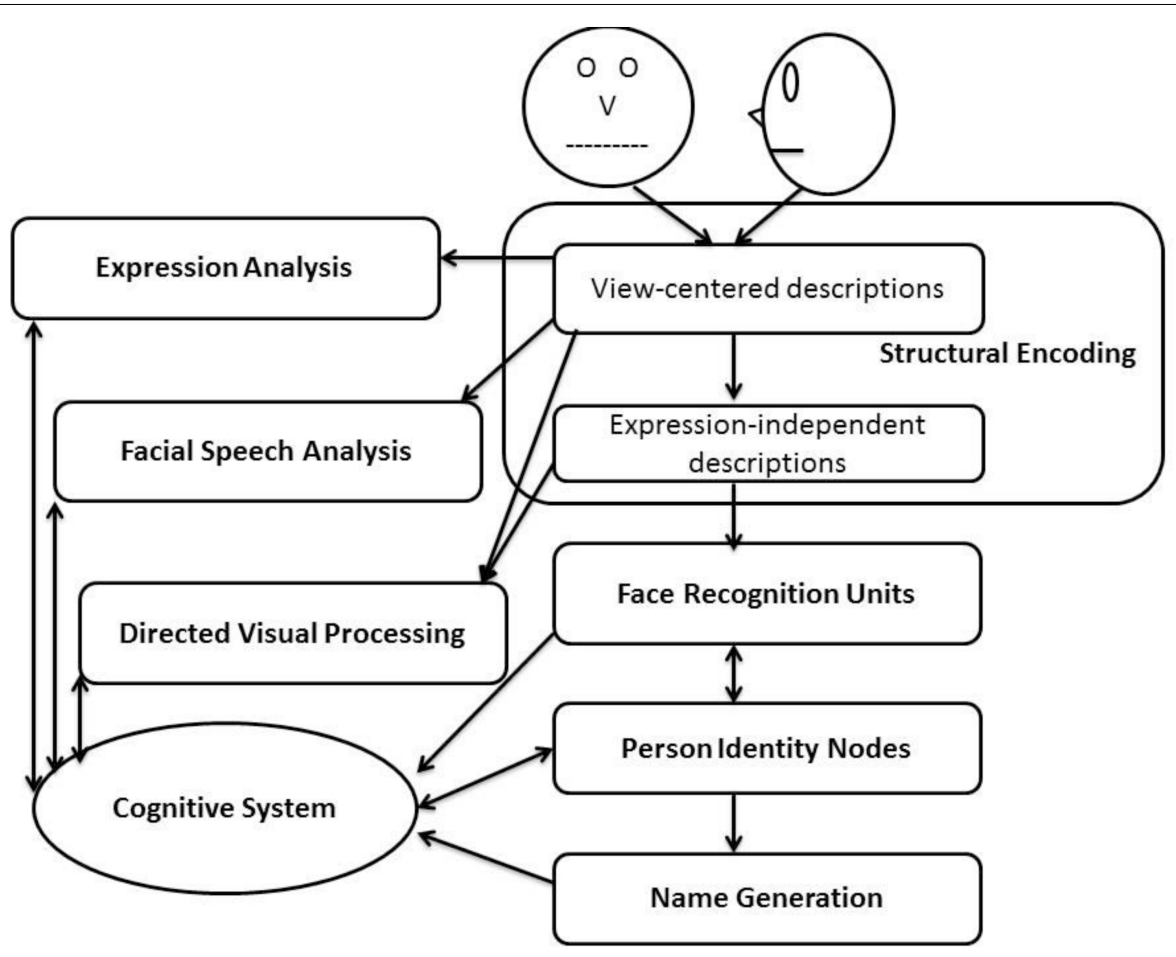

FIGURE 1 | The functional model of familiar face recognition (re-drawn from Bruce and Young, 1986).

how and what kind of information from the mid-level visual processing would be funneled into the separate routes of the part-based processing and holistic/configural processing.

Regarding the first question, McKone had suggested prosopagnosic patients could rely on part-based recognition system to detect presence of faces, whereas damage to the holistic/configural face recognition systems would prevent them from recognizing familiar faces. Consistent with this interpretation, a number of studies had demonstrated that indeed prosopagnosia patients can detect faces, and at the same time showed impairments in holistic processing (e.g., Avidan et al., 2011). Regarding the second question, it seems that there can be no clear-cut temporal separation between part-based processing and holistic processing because holistic processing occurs rapidly within $50 \mathrm{~ms}$ after the onset of face stimuli (Richler et al., 2009). In other words, findings from previous behavioral studies have offered little evidence for the temporal separation between holistic processing and part-based processing. Finally, regarding the third question, while making assumptions about the separate, parallel routes of processing for her dual-route model, McKone failed to give sufficient details to subject the proposed model to empirical enquiries, and factors that putatively would give rise to holistic processing were also left unspecified. Regardless whether holistic/configural face recognition system is unitary or comprises separate subcomponents, it is important to account for how the product of mid-level vision would channel face processing into part-based recognition systems and holistic/configural face recognition systems in general, and how exactly holistic/configural processing can be derived from

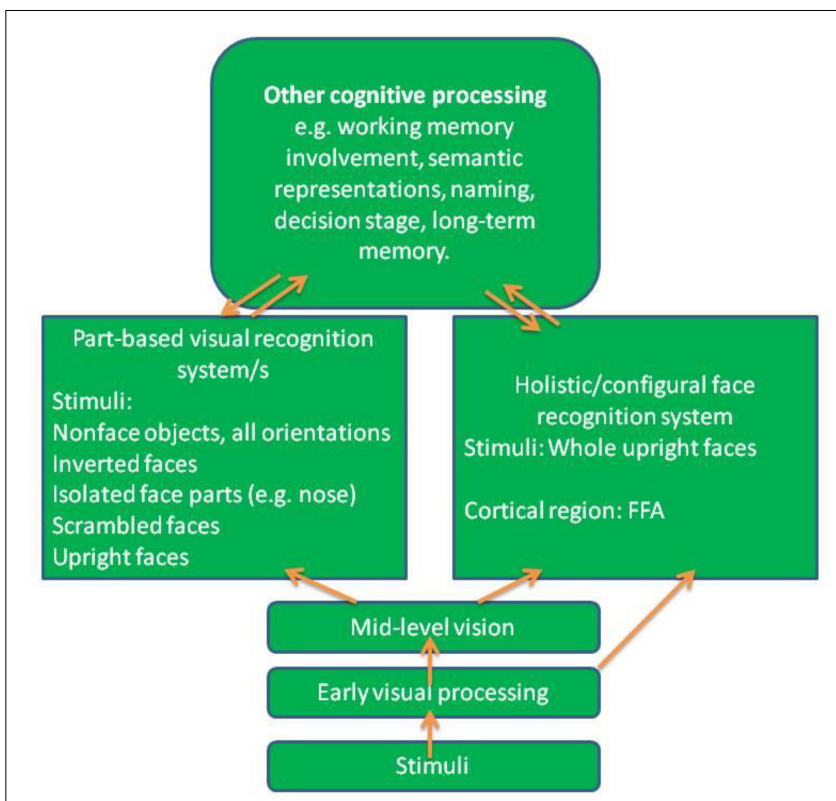

FIGURE 2 | Early visual processing, mid-level vision and long-term memory all would affect holistic processing with the exception of part-based visual recognition system (re-drawn from McKone, 2010).

the mid-level vision in particular. One likely candidate for the product of mid-level vision would be a representation akin to the face template, and many studies have suggested that holistic 
processing arises from a face template (Gauthier et al., 2010; Richler et al., 2012; Richler and Gauthier, 2014). However, there has been very little research to examine directly the relationship between a face template and holistic processing.

According to Richler et al. (2012), many putative mechanisms can be hypothesized to be potential instigator for holistic processing, including global face templates, representation of spatial relations between parts, inflexible attentional weightings on parts and parallel coactive processing, etc. It would require a formidable amount of studies to test the validity of each of these hypothesized mechanisms. Even though many researchers have indicated that face parts are recognized holistically as a gestalt based on a face template (e.g., Young et al., 1987; Tanaka and Farah, 1993; Farah et al., 1998), the idea of face template remains a relatively vague concept for understanding face processing. The following section discuss the relationship between face template and holistic processing.

\section{Face Template and Holistic Processing}

As mentioned above, many researchers seem to have adopted the view that holistic processing arises as a consequence of extensive experiences with a face template (Tanaka and Farah, 1993; Rossion and Boremanse, 2008; Chua et al., 2014). Rossion and Boremanse (2008), for example, found that the composite face illusion, where holistic processing is indexed by the composite face effect (CFE), was equally strong when faces were presented at $0^{\circ}-60^{\circ}$ on a fronto-parallel picture plane, then dropped off at $90^{\circ}$ and maintained up to $180^{\circ}$. They contended that faces have been processed holistically at angular rotation less than $90^{\circ}$, and the dramatic drop of composite effect when faces were rotated beyond $90^{\circ}$ suggests that holistic face perception may have resulted from experience-driven template of upright faces (Rossion, 2013).

However, other researchers have suggested that face parts are "glued" together to form a unitary perceptual representation or "face template" (e.g., Young et al., 1987; Tanaka and Farah, 1993; Farah et al., 1998). Such usage of terminology and descriptions can sometimes be confusing and misleading because it leaves very little room for distinction between face template and holistic processing. As a more useful alternative, consider Tsao and Livingston's (2008) proposal that face detection is the first step in face processing, especially when the face is embedded in a complex visual scene. Importantly, their proposal implicates that a face template can be based on the simple T-shape configuration comprising a pair of eyes above a nose and the nose above a mouth. Neurophysiological evidence has led credence to such a notion of face template, where many studies of single-cell recording have found that face cells require an intact face configuration as effective eliciting stimulus, and are not selectively responsive to facial features (Bruce et al., 1981; Desimone et al., 1984; Kobatake and Tanaka, 1994; Tsao et al., 2006). These studies demonstrated the dissociation between an intact face configuration (i.e., face template) and facial features comprising the configuration.

In addition, activation in inferior temporal cortex for faceselective neurons begins firing $80-130 \mathrm{~ms}$ after stimulus onset (Bullier, 2001). An ERP study also reported that the time window for face detection was about $130 \mathrm{~ms}$ (Jacques and Rossion, 2006). Furthermore, according to Bentin et al. (1996), an inverted face and partial facial features (e.g., nose and lips) had a delayed N170 component in comparison to an upright face, which implies different time courses for holistic processing and part-based processing. However, there have been relatively few behavioral studies that investigate the time course difference between holistic processing and part-based processing, and in particular it is unclear whether holistic processing arises from a face template directly (Chua et al., 2014).

It should be noted that there are at least two different notions of face template being advocated in the existing literature. On one hand, face template and holistic processing are treated as synonymous and have been used interchangeably; on the other hand, face templates strictly entail the T-shape configuration and first-order relational properties within a face.

\section{Holistic Processing and Sensitivity to Configuration}

Yin's (1969) study was the first that demonstrates the disproportionate inversion effect with faces. During both the perception and memory phase of his study, Yin's participants showed better performance with upright faces than with inverted faces (Figure 3). In a sharp contrast, inversion had little effect on non-face objects such as houses and airplanes. Based on these findings, Yin suggested that perception of human face is strongly influenced by orientation, due to the fact that faces were processed holistically and inversion disrupts holistic processing and results in poorer performance on inverted faces.

Subsequently, Diamond and Carey (1986) proposed that three conditions need to be met to lead to performance decrement for the inversion effect: first, members of the group of stimuli must share the same first-order configuration. Second, differences between individual members are on the basis of second-order relational features. Finally, participants who are experts can distinguish these members of the group relying upon such second-order relational information. As a case to support their conjecture, Diamond and Carey showed in their study that dog experts exhibited inversion effect in recognition of breeds they were most familiar with (and hence showing expertise).

To further examine holistic processing in faces and objects, Tanaka and Farah (1993) used a part-whole task and showed that people can better recognize a face part (e.g., nose) in the context of a whole face than in isolation. In contrast, they found no part-whole differences when participants were asked to recognize other kinds of object such as scrambled parts, inverted faces, and houses.

Tanaka and Farah argued that their findings suggest people recognize faces by engaging in holistic processing. Moreover, in a subsequent study, Tanaka et al. (1998) argued that children develop holistic processing for faces by the age of 6 years old, although more generally, face recognition seems to mature at around 12 years of age (Johnson and Morton, 1991; Mondloch et al., 2002). Studies in perceptual grouping also suggest that around 5-6 years old, children have developed perceptual organization capability comparable to that for adults (Hadad 


\section{Inversion Effect}

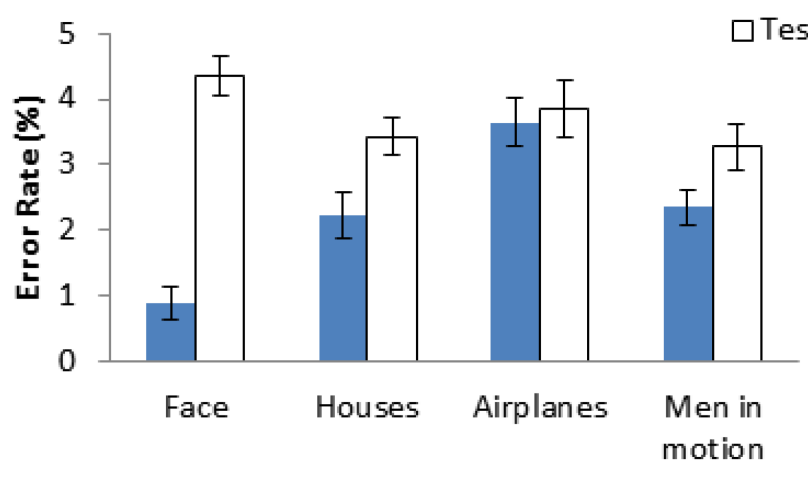

\section{Categories}

FIGURE 3 | In Yin (1969) study, there was a significant difference in performance between upright and inverted faces; in contrast, no significant differences were found for objects of non-face categories. The error bar indicates the mean of \pm 1 standard error. The figure was adapted and modified from Yin (1969).

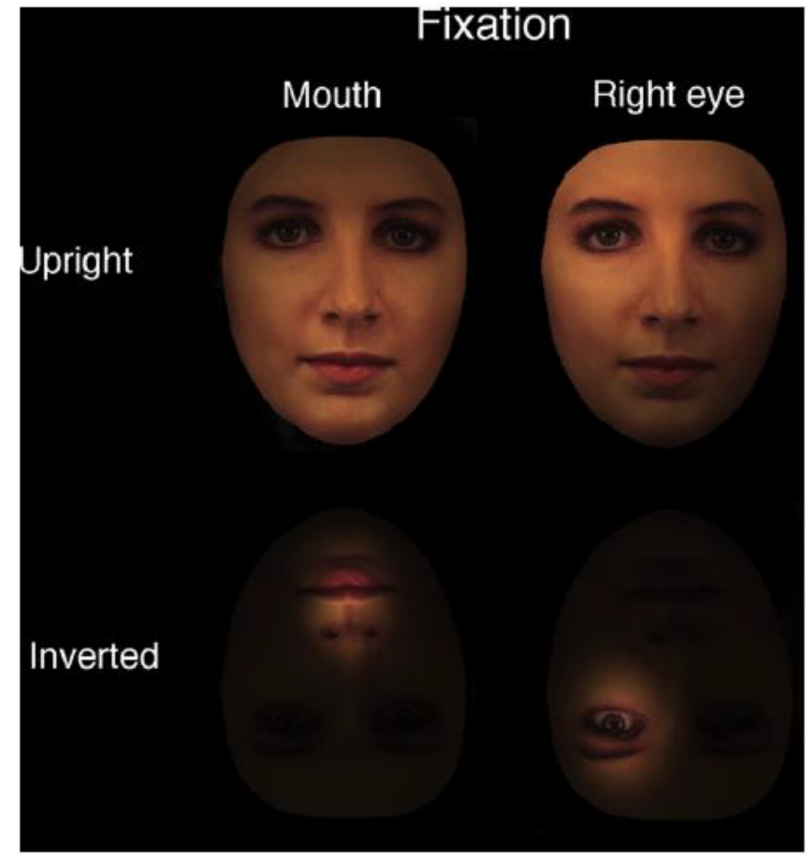

FIGURE 4 | According to the perceptual field hypothesis, participants perceive an upright face as a whole face, rather a collection of local features. In contrast, participants perceive inverted faces as local features, rather than a whole face. The figure was adapted from Rossion (2009) with permission.

and Kimchi, 2006). It should be noted, however, it is unclear whether holistic/configural face recognition system is different from part-based recognition systems because there is a lack of relevant evidence to support the dual-route model based on developmental studies.

Although disruption of holistic processing has been hypothesized to account for inversion effect, it is less clear

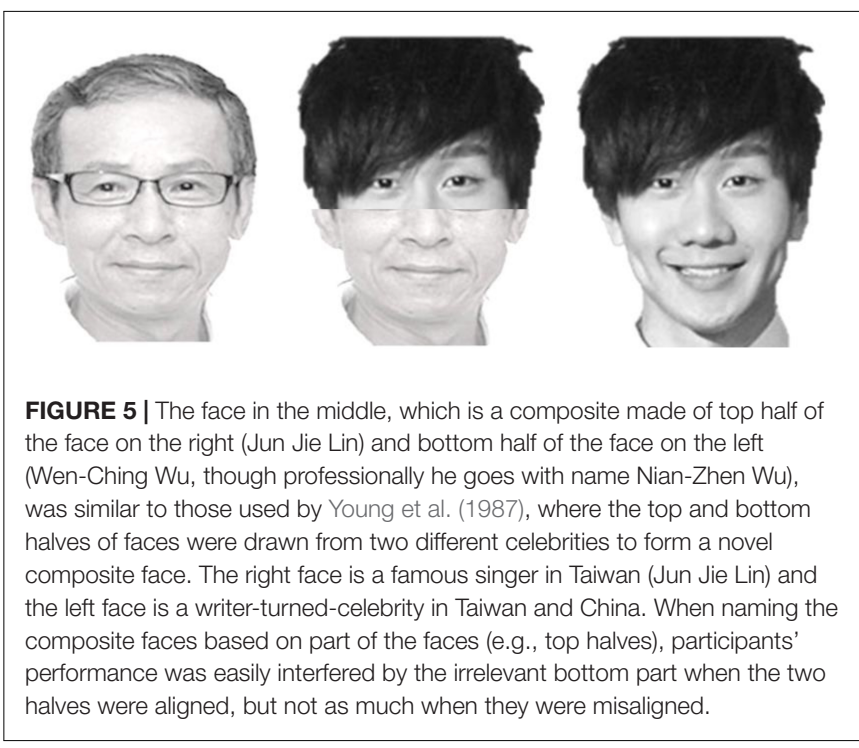

what exactly may have taken place during inversion that would lead to such disruption. One possible candidate for the underlying mechanism is the perceptual field hypothesis Rossion (2009) proposed, which would explain how inversion impairs holistic processing in the following manner: upon seeing an upright face, observers presumably would expand their perceptual field to the fullest extent in order to perceive the whole face (Tanaka and Gordon, 2011; Rossion, 2013). In contrast, when seeing an inverted face, observers would perceive something less than a whole face, which likely is composed of a constellation of local facial features due to the contraction of perceptual field (Figure 4). It is interesting to note that McKone (2010, p. 284) also mentioned in the passing a similar account for holistic processing arisen from "a big receptive field" being applied to the entire face. 


\section{Composite Effect and Composite Face Illusion}

Young et al. (1987) developed another paradigm - the composite faces-to demonstrate holistic processing of faces (Figure 5). Although they used the term "configural" rather than "holistic," to avoid conceptual confusion, it would consider their effect as demonstrating holistic, rather than configural, processing because composite faces involve altering both featural and configural information of a face simultaneously. In their experiments, Young et al. created composite faces of celebrities in that the top and bottom half parts of different celebrities were joined to form novel faces. Participants' performances in naming the celebrity based on top half of the face were severely compromised by the creation of composite faces. Young et al. concluded that participants recognized famous faces by means of holistic processing. Following Young et al.'s (1987) study, composite effect is not obtained with objects or non-face stimuli (Robbins and McKone, 2007), in addition to the fact that they were little affected by inversion.

Following Young et al.'s study, Hole (1994) further demonstrated that unfamiliar faces also were affected by the irrelevant parts. In each trial of their experiment, a pair of faces was simultaneously presented, which was different from the naming task used by Young et al. (1987). Despite the difference in task requirement, Hole's findings were consistent with those reported by Young et al. (1987). Taken together, these studies suggested that both familiar and unfamiliar faces are processed holistically rather than in a piecemeal manner (Young et al., 1987; Tanaka and Farah, 1993; Hole, 1994).

Ever since the pioneering work by Young et al. (1987) and Hole (1994), many subsequent studies have adopted the composite face task to assess the failure of selective attention to the target part, which was interfered by the irrelevant parts as a result of combining halves from different faces. Participants cannot focus selectively on the specific part of a face (e.g., the top half) while at the same time ignore the irrelevant part (e.g., the bottom half), implicating that face recognition is achieved via integrated holistic processing, rather than via a piecemeal process where each facial feature was processed and then combined. However, as Rossion (2013) pointed out, there is a difference between composite effect and composite face illusion. Specifically, he argued that participants actually perceived the composite face as a new face, in the sense that facial features had been modified perceptually rather than being perceived veridically (called composite face illusion). As a result, holistic processing for upright faces not only affects sensitivity of configuration among features but also on the features themselves. Maybe, it is better to the term composite face illusion rather than composite face effect.

\section{A Note on Conceptual Clarification and the General Method}

Before describing the experiments proper, I would like to clarify the conceptual definitions of a number of popular notions to avoid the possible confusions (cf. Richler et al., 2012). In so doing, it is necessary to give a description of the General Method used here.

Generally speaking, holistic processing of a face refers to integral processing of all parts of a face as a whole in that processing one part of the face would inevitably entail processing another part of the face. More specifically, there are two important and yet distinct aspects regarding the notion of holistic processing. The first aspect concerns the perceptual integrality just mentioned. The second aspect concerns the strong and inherent dependency of orientation in face processing where holistic processing is more evident and robust for an upright than for an inverted face. Part-based, featural or component processing of a face, on the other hand, typically involves manipulation or alteration of face components while keeping intact the configuration and the metrical distances between components (Mondloch et al., 2002). Finally, configural processing of a face entails manipulation of inter-distance between face components while keeping intact the components (Gauthier and Tarr, 2002; Mondloch et al., 2002).

As Rossion (2008) pointed out, although configural processing and holistic processing can be dissociated conceptually, they are confused inherently in the practice of empirical enquiry. While most researchers would agree that holistic processing for face recognition entails simultaneously integration of multiple features of a face into a single representation (Rossion, 2008), there remains the question whether configural processing should be treated as part of holistic processing. For some researchers, it has been common to associate changes in spacing between facial features with holistic processing (Gauthier and Tarr, 2002; McKone, 2010). For instance, Gauthier and Tarr (2002) treated the term "holistic" as a superordinate concept and encompassed a variety of effects, such as holistic-configural, holistic-inclusive, and holistic-contextual effects. In the view, I would like to define holistic processing as the integration of component and configural processing, which is similar to the "holistic-contextual effect" proposed by Gauthier and Tarr (2002).

Previous studies have used definitions that in the view were not completely compatible with one another (Richler et al., 2012). Robbins and McKone (2007), for example, used the term "configural/holistic processing" to suggest that configural processing is synonymous to holistic processing, and it was not necessary to make fine distinction between them given the current state of research findings and evidence (see also McKone, 2010). However, Mondloch et al. (2002) study had argued earlier for the distinction between configural and holistic processing, where configural processing focuses on spatial (metric) relations between and among face parts, and hence is different from holistic processing, which focuses on the integration of face as a perceptual whole or gestalt. Moreover, Hole (1994) used the term, "configurational factor," to describe the compositeface effect, whereas Gauthier and her colleagues have over the years used the term of "holistic processing" in an all-embracing manner to refer to the processing underlies the composite-face effect. Together these examples highlight the fact that the term "holistic processing" has not been used in a completely coherent and consistent manner in the literature, where not only it has different meanings for different researchers but also may refer 


\section{Complete Design}

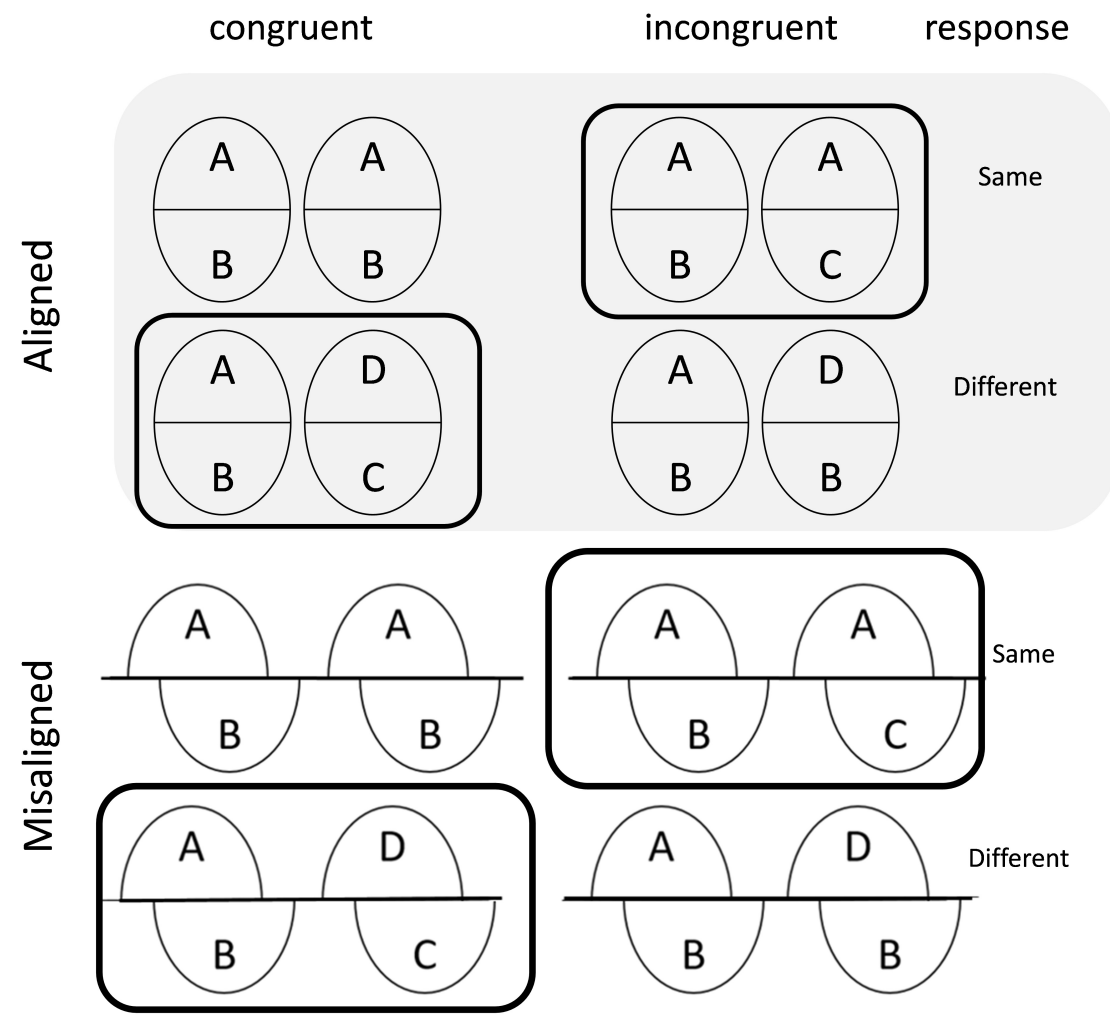

FIGURE 6 | Many prior experiments had used the partial design (black frame) to demonstrate holistic processing, but the irrelevant part was always different. Although it showed slower performances for same trials, it was possible that the poorer performance had resulted from congruency effect, rather than holistic processing. In the complete design, showed at the figure could control the confounding variable and avoid an erroneous conclusion. The figure was redrawn from Gauthier and Bukach (2007).

to different tasks and different associated effects (Richler et al., 2011).

Partial versus complete design of composite face task. Gauthier and Bukach (2007) had proposed to use what they called complete design to replace the traditional composite task, also called the partial design, used in many previous studies based on two reasons. First, some researchers had indicated that only the results from the same trials in the partial design were computed (Robbins and McKone, 2007; Rossion, 2013) while ignoring completely the results from different trials. Gauthier and colleagues argued that the results should include both the same trials and different trials because results from both kinds of trials are meaningful for explaining the composite illusion (Gauthier and Bukach, 2007; Richler and Gauthier, 2014). When different trials are ignored in the partial design, it is impossible to predict whether perceiving information from irrelevant parts facilitate or interfere performance when relevant parts are different. In addition, when the same trials in the congruent condition and different trials in the incongruent condition were missing, there was not enough information to predict how irrelevant part affects holistic processing in those conditions. When the responses for relevant and irrelevant part are the same (i.e., both "same" or both "different"), these trials are in the congruent condition. In contrast, when the responses are different (i.e., one "same" and the other "different" or vice versa), these trials are in the incongruent condition.

The second and perhaps more critical reason is that the partial design is subject to response biases from individual participants (Richler and Gauthier, 2014) because they tend to respond "same" in the upright face condition than in the inverted face condition (Wenger and Ingvalson, 2003; Richler and Gauthier, 2014) and in the aligned condition than the misaligned condition (Gauthier and Bukach, 2007). To rule out the potential problems, Gauthier and Bukach (2007) had proposed that holistic processing should be assessed and measured in terms of a congruency effect (i.e., the difference in performance between the congruent and incongruent conditions) and the dependent variable is sensitivity $\left(d^{\prime}\right)$, which is a difference score $\left(Z_{n}-Z_{s n}\right)$ based on signaldetection theory (SDT) (where $n$ denotes "noise" condition, and $s n$ denotes "signal + noise" condition) (Green and Swets, 1966). The performance in sensitivity $\left(d^{\prime}\right)$ is expected to be better on the congruent trials than on the incongruent trials in the aligned 
(or upright) condition and the magnitude of congruency effect is reduced in the misaligned (or inverted) condition. Moreover, the magnitude of congruency effect with aligned (or upright) faces is expected to be greater than that with misaligned (or inverted) faces. Theoretically speaking, it is appropriate to use the complete design in the composite task.

How can congruency effect be measured? Referring to Figure 6, the complete design involves congruent and incongruent conditions. The congruent condition means that the target and the irrelevant part are both same or both different. In contrast, the incongruent condition means that those are inconsistent. If the present study would calculate congruency effect in aligned condition, the result was that $d^{\prime}$ of congruent trials minus $d^{\prime}$ of incongruent trials (Gauthier and Bukach, 2007). Some researchers suggested inverted faces are the same as misaligned face because of impairment for configural processing. On the other hand, the irrelevant parts are always different in the partial design (see black framed panels in Figure 6). Moreover, the partial design only calculates accuracy or reaction time between same trials of aligned and misaligned condition and neglect altogether data from different trials.

Moreover, Zhao et al. (2015) recently suggested alignment effect (or interaction between alignment and congruency effect) can even occur for no-face stimulus (e.g., line pattern) as long as it possesses salient Gestalt properties such as connectedness, closure, and continuity between parts. Bases on these observations, Zhao et al. (2015) argued that alignment effect could be achieved not only via a top-down route from becoming experts, but also via a bottom-up route of relying on object-based information.

In addition, McKone et al. (2013) proposed that inverted faces always should be included such that a pure measure of holistic effect can be achieved to the extent that there is an absence of holistic processing with inverted faces. Based on these suggestions, inverted condition could be a better measure to detect holistic processing than the manipulation of alignment. Therefore, in the experiments reported here, the adopted dependent variable was the difference in congruency effect between upright and inverted conditions using the complete design.

Besides the definition of holistic processing for the dependent variable, the present study also measured the performances of the part-based processing as the dependent variable in each experiment. According to McKone (2010), the part-based processing includes upright and inverted faces. Therefore, the definition of part-based processing included the performances of the upright and inverted conditions. In other words, the performances of part-based processing were the sensitivity for the upright and inverted face trials including the congruent and incongruent trials. If the performances of the participants are above chance, it means the part-based processing is observed.

\section{EXPERIMENT 1}

The issue of time course also concerns whether holistic processing may exist at a glance, and when it would disappear after a longer delay. Richler et al. (2009) proposed that holistic processing emerged very rapidly because they found that participants' performance was above chance and did not depend on exposure time (see Figure 7). Note, however, their prediction was inconsistent with Hole's (1994) findings, where he failed to find evidence of holistic processing at the duration of 2,000 ms; however, Richler et al. (2009) did not examine whether holistic processing occurs and still exists at the duration beyond $800 \mathrm{~ms}$.

It should be noted, however, that there were inconsistent results regarding whether holistic processing was obtained with a relatively short term of duration. Therefore, in Experiment 1, a range of relatively brief exposure duration, namely 100, 200, and $500 \mathrm{~ms}$, was manipulated to see whether or not can replicate these results for separation between holistic processing and part-based processing with these brief durations. In contrast, in Experiment 2 , it manipulated relatively long exposure duration to see whether holistic processing still can be obtained with 1000 and $2000 \mathrm{~ms}$. There has been a controversial issue whether holistic processing was obtained at $2000 \mathrm{~ms}$. On one hand, Hole (1994) was unable to observe holistic processing at $2000 \mathrm{~ms}$, but Richler et al. (2011) predicted that holistic processing should be obtained at $2000 \mathrm{~ms}$ according to their hypothesis (see Figure 7). In order to recreate the experimental setup as closely to that used by Hole (1994) as possible, a pair of faces was presented diagonally in each trial to reduce the possibility that participants might adopt a feature-byfeature comparison strategy to cope with the task. In addition, the whole study practically adopted a between-participant design and separate the two ranges of exposure duration in Experiments 1 and 2 as a means to provide a more conservative test of time course for part-based and holistic processing.

\section{Methods \\ Participants}

Eighteen participants (9 male) from National Chung Cheng University participated in Experiment 1, and none of them had been exposed to the face composite task in the past 3 months. All participants had normal or corrected to normal vision, and each received a monetary payment of NTD $\$ 120$ for their participation. It took about $55 \mathrm{~min}$ for participants to complete the experiment. Data from one participant had to be excluded because his overall performance was below chance. Likewise, the data from another participant was excluded because the performance was worse than two standard deviations from the mean. Participants were recruited in accordance with approval of the Research Ethics Committee of National Chung Cheng University, ChiaYi, Taiwan (No. CCUREC104082101). All participants had completed the informed consents before the experiment.

\section{Stimuli}

For face stimuli, the present study first created 32 different Asian face images with equal number of males and females using FaceGen 3.1 (Singular Inversions, Canada). Half of them, 8 males and 8 females, were chosen as the relevant set, and the remaining half, also 8 males and 8 females, was chosen as the irrelevant set. The top halves from the relevant set were randomly paired with the bottom halves from the irrelevant set for constructing face composites, in accordance with the requirement of complete 

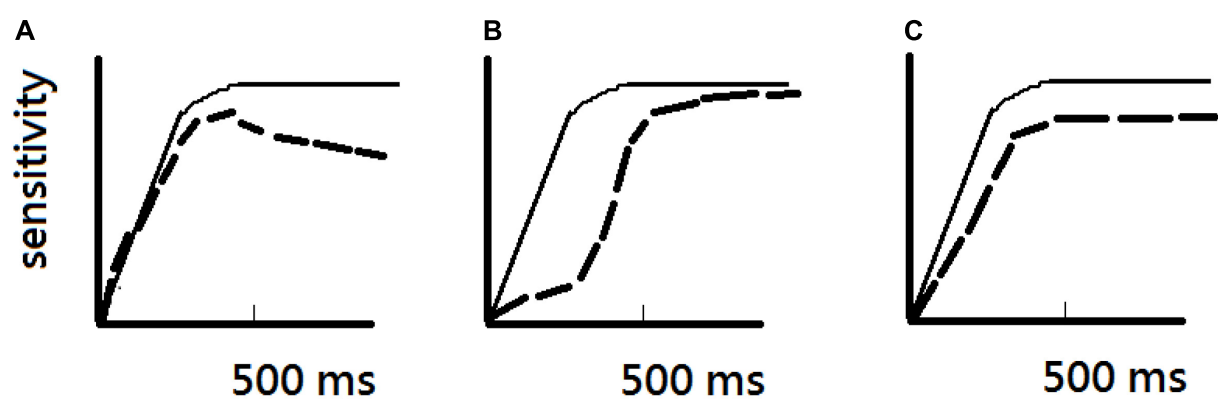

congruent

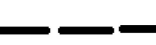

incongruent

FIGURE 7 | Richler et al. (2009) proposed three possibilities and their findings supported predictions depicted in (C). Holistic processing, measured in terms of difference in performance between congruent and incongruent conditions, occurs at all exposure time when performances are above-chance performance. The figure was redrawn from Richler et al. (2009). (A) Illustrates that holistic processing occurs after a minimum of exposure time and increases as exposure duration was increased. (B) Illustrates that holistic processing is larger when exposure duration is limited. (C) Illustrates that holistic processing occurs without interference of exposure duration.

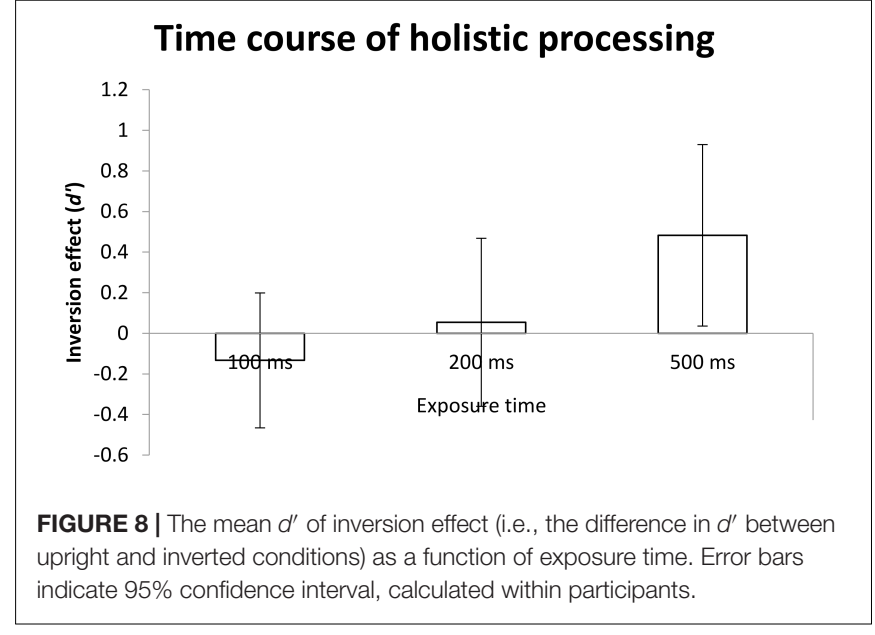

design. Specifically, there were 16 faces for each of the four face composites designated as "A (top)/B (bottom)," "A (top)/C (bottom)," "D (top)/C (bottom)," and "D (top)/B (bottom)" (see Figure 6).

Each face image could be encircled by an (invisible) square with 75 pixels on each side. When presented on the display screen, each face was about $3.38 \mathrm{~cm}$ in width and $4.58 \mathrm{~cm}$ in height, extending a visual angle of about $3.01^{\circ} \times 3.6^{\circ}$ at a viewing distance of approximately $45 \mathrm{~cm}$. An overextended white line was overlaid horizontally in the mid-section of each face to clearly demark the top and bottom half of the face. The line was of $7.79 \mathrm{~cm}$ in length and $0.14 \mathrm{~cm}$ in height, extending a visual angle of $6.14^{\circ} \times 0.11^{\circ}$.

\section{Design and Procedure}

As mentioned above, in Experiment 1, the design manipulated a relatively brief range of exposure time of 100, 200, and $500 \mathrm{~ms}$, as a within-participant variable. As in Experiment 1, it adopted the complete design (Figure 6) and computed differences in congruency effect between upright and inverted

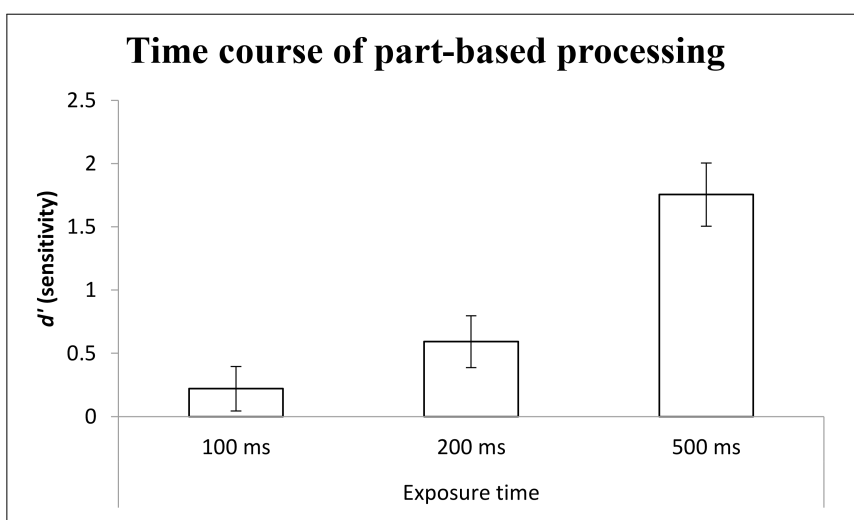

FIGURE 9 | The mean $d^{\prime}$ (sensitivity) for indexing part-based processing in each condition as a function of exposure time. Error bars indicate 95\% confidence interval, calculated within participants.

faces for each participant as the dependent variable (see section "A Note on Conceptual Clarification and the General Method").

Each composite face was presented either upright or inverted for a total of 384 trials, comprising equal number of congruent and incongruent trials in accordance with the design used in Experiment 1 . The upright face and inverted face conditions were separated into different blocks, the order of administration was counterbalanced across participants. In each trial, a "+" for fixation was first shown at the center of the display for $500 \mathrm{~ms}$, followed by the presentation of a pair of composite faces, diagonally presented for one of the three durations, 100, 200 , or $500 \mathrm{~ms}$, where exposure time was manipulated as a within-participant variable. When the face stimuli was shown for the pre-designated exposure duration, a mask was presented for $200 \mathrm{~ms}$. The manipulation of face orientation also was done as a within-participants variable by including two blocks of trials, one block with upright faces and the other with inverted faces. The order of the two blocks was counterbalanced across participants. 


\section{Results}

Mean percentage of correct identification, expressed in terms of hit rate (HR) and correction rejection (CR), of the top halves of upright and inverted conditions are shown in Table 1. The results show the same set of analyses as done in Experiment 1 as follows:

\section{Holistic Processing}

One-way measure ANOVA was conducted with exposure time $(100,200$, and $500 \mathrm{~ms})$ as the within-participant factor. As shown in Figure 8, the main effect of exposure time was not significant, $F(2,32)=3.08, M S E=1.70$, $p>0.05, \eta_{\mathrm{p}}^{2}=0.161$, indicating no difference in the magnitude of holistic processing as a function of exposure duration.

In terms of planned comparisons, one-sample $t$-tests and used Holm-Bonferroni method to control the family wise error rate $\left(\alpha_{B}=0.017, \alpha_{B}=0.025, \alpha_{B}=0.05\right)$. were performed to test whether holistic processing would occur at each level of exposure duration. The results reveals that holistic processing was not observed at the level of two shorter exposure durations of $100 \mathrm{~ms}$ and $200 \mathrm{~ms}$, $t \mathrm{~s}<1$. However, holistic processing was obtained at exposure duration of $500 \mathrm{~ms}$ marginal significantly $(M=0.48)$, $t(16)=2.29, p=0.036>\alpha_{\mathrm{B}}=0.017,95 \% \mathrm{CI}=[0.04,0.93]$.

\section{Part-Based Processing}

The same one-way ANOVA was conducted with exposure time as the within-subjects factor, and $d^{\prime}$ was the dependent measure for part-based processing. The main effect of exposure time was significant, $F(2,32)=103.63, M S E=10.9, p<0.001, \eta_{\mathrm{p}}^{2}=0.87$. Post hoc comparisons with Bonferroni correction $\left(\alpha_{\mathrm{B}}=0.017\right)$ revealed that performance at $500 \mathrm{~ms}$ was better than those at 100 and $200 \mathrm{~ms}$, $\left.p s<0.01<\alpha_{B} s=0.017\right)$, and performance at $200 \mathrm{~ms}$ in turn was better than that at $100 \mathrm{~ms}\left(p<0.01<\alpha_{\mathrm{B}} \mathrm{s}=0.017\right)$. The results suggested that evidence of part-based processing

TABLE 1 | Descriptive statistics for each condition in Experiment 1.

\begin{tabular}{|c|c|c|c|c|c|}
\hline \multirow[t]{2}{*}{ Orientation } & \multirow[t]{2}{*}{ Congruent } & \multirow[t]{2}{*}{ Response } & \multicolumn{3}{|c|}{ Exposure duration } \\
\hline & & & $100 \mathrm{~ms}$ & $200 \mathrm{~ms}$ & $500 \mathrm{~ms}$ \\
\hline \multirow[t]{6}{*}{ Upright } & Congruent & $H R$ & $0.50(0.21)$ & $0.55(0.21)$ & $0.86(0.13)$ \\
\hline & & $\mathrm{CR}$ & $0.59(0.21)$ & $0.66(0.25)$ & $0.86(0.11)$ \\
\hline & & $d^{\prime}$ & $0.24(0.58)$ & $0.78(1.04)$ & $2.42(0.69)$ \\
\hline & Incongruent & $H R$ & $0.40(0.21)$ & $0.46(0.22)$ & $0.72(0.16)$ \\
\hline & & $\mathrm{CR}$ & $0.65(0.20)$ & $0.67(0.21)$ & $0.74(0.19)$ \\
\hline & & $d^{\prime}$ & $0.18(0.64)$ & $0.44(0.53)$ & $1.57(0.63)$ \\
\hline \multirow[t]{6}{*}{ Inverted } & Congruent & $\mathrm{HR}$ & $0.50(0.21)$ & $0.66(0.17)$ & $0.86(0.14)$ \\
\hline & & $\mathrm{CR}$ & $0.58(0.25)$ & $0.63(0.17)$ & $0.67(0.23)$ \\
\hline & & $d^{\prime}$ & $0.38(0.73)$ & $0.83(0.52)$ & $2.00(0.91)$ \\
\hline & Incongruent & $\mathrm{HR}$ & $0.53(0.22$ & $0.62(0.17)$ & $0.84(0.15)$ \\
\hline & & $\mathrm{CR}$ & $0.54(0.22)$ & $0.60(0.18)$ & $0.66(0.20)$ \\
\hline & & $d^{\prime}$ & $0.18(0.39)$ & $0.57(0.45)$ & $1.71(0.76)$ \\
\hline
\end{tabular}

Means of hit rate (HR) and correct rejection (CR) for upright and inverted face condition at each level of exposure time. Standard deviations are shown in parentheses. emerged at about after $100 \mathrm{~ms}$ after stimulus onset and increased as more exposure time was available for further processing.

In terms of planned comparisons, as shown in Figure 9, the results were again used one sample $t$-test and used HolmBonferroni method to control the family wise error rate $\left(\alpha_{B}=0.017, \alpha_{B}=0.025, \alpha_{B}=0.05\right)$. The performances were above chance at all three levels of exposure time $\{100 \mathrm{~ms}: t(16)=2.65$, $p=0.017<\alpha_{\mathrm{B}}=0.05, M=0.22,95 \% \mathrm{CI}=[0.04,0.40] ; 200 \mathrm{~ms}:$ $t(16)=6.13, p<0.001<\alpha_{\mathrm{B}}=0.025, M=0.59,95 \% \mathrm{CI}=[0.39$, 0.80]; 500 ms: $t(16)=14.87, p<0.001<\alpha_{B}=0.017, M=1.76$, $95 \% \mathrm{CI}=[1.51,2.01]\}$. These results again indicate that partbased processing was in place very early on in processing faces after their presentation.

\section{Discussion}

The results of Experiment 1, which offered strong support to the conjecture of separation between holistic processing and partbased processing, where the results also suggested that holistic processing occurred after part-based processing occurred. That is, the $d^{\prime}$ results for each condition showed clear evidence for an early onset of part-based processing, whereas the inversion effect as evidence for holistic processing did not emerge until a longer exposure duration of $500 \mathrm{~ms}$. These findings support to the time course of face processing depicted in Figure 7A. Alternatively, there is another possibility to explain the results where holistic processing and part-based processing occur simultaneously, but holistic processing may be prone to the disruption due to low stimulus discriminability as may well be the case in the face stimuli used in Experiment 1 because the means of the performances in the experiment were in general lower than the means of the studies from Richler et al. (2009, 2011). Even though the congruency effect between congruent and incongruent trials as Richler et al. (2009) did, the results still could not find the significant congruency effect at 100 and $200 \mathrm{~ms}$ ( $t$ s $<1.43$ or ps $>0.1$ ), but did find a significant congruency effect at $500 \mathrm{~ms}$, $t(16)=5.17, p<0.001$.

\section{EXPERIMENT 2}

As noted earlier, there has been an important and yet controversial issue regarding the time course of face processing. In order to examine whether the holistic processing of faces would sustain beyond the initial moment of detecting their presence, the present experiment used a simultaneous composite face task, where a pair of face stimuli were exposed for relatively lengthy duration of 500, 1000, or $2000 \mathrm{~ms}$, respectively. With these durations, Hole (1994) had indicated that there was no difference between the upright and inverted faces at the long duration of $2000 \mathrm{~ms}$. His result was inconsistent with the predictions made by Richler et al. (2009) (see Figure 7). However, Richler and her colleagues had not endeavored to demonstrate that holistic processing occurred after or beyond $800 \mathrm{~ms}$. Therefore, Experiment 2 was designed to examine this controversy (Richler et al., 2009).

In order to have maximum opportunity to replicate Hole's (1994) original findings, the present experiment adopted to 


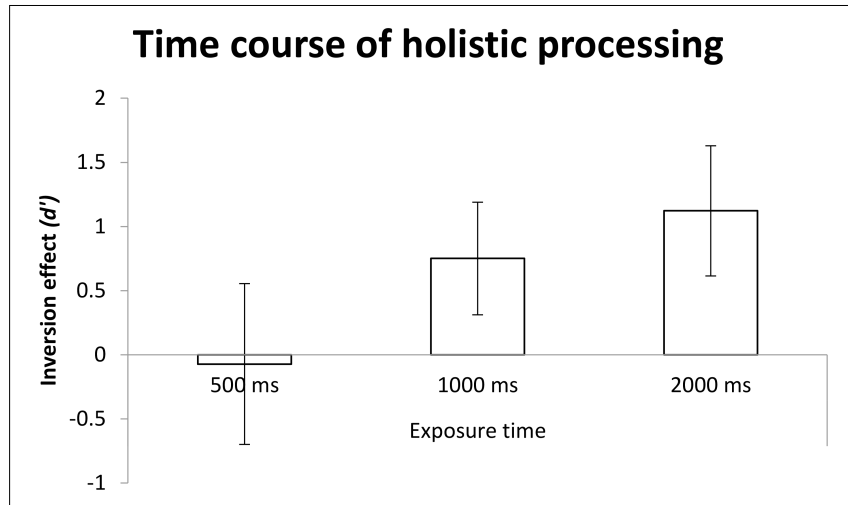

FIGURE 10 | The mean $d^{\prime}$ for dependent variable as a function of exposure time. Error bars indicate 95\% confidence interval, calculated within participants.

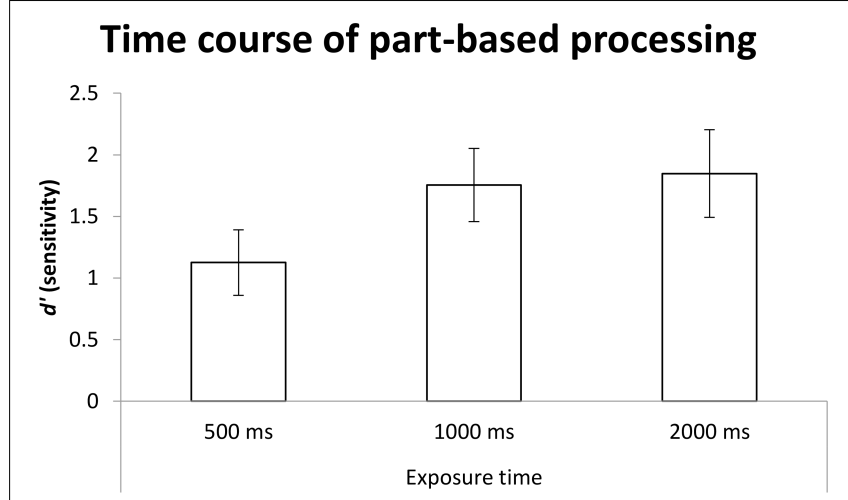

FIGURE 11 | The mean $d^{\prime}$ for sensitivity as a function of exposure time. Error bars indicate $95 \%$ confidence interval, calculated within participants.

display two face images in each trial diagonally to circumvent participants' use of a feature-by-feature comparison strategy. In a pilot test, it was very difficult for participants to view two face images simultaneously when they were presented briefly for $500 \mathrm{~ms}$, therefore adjusted stimulus size to make the face images easier to view and removed the mask that comes after showing two composite faces in Experiment 1, as Hole (1994) did. In addition, Experiment 2 adopted between-participant design regarding the manipulation of exposure duration to provide a more conservative test of the issue at hand.

\section{Methods}

\section{Participants}

Fifty-eight college students from the National Chung Cheng University in Chiayi County, Taiwan, participated in the experiment. Of them, 21 (6 male, 15 female) were assigned to the exposure condition of $500 \mathrm{~ms}, 17$ (6 male, 11 female) to $1000 \mathrm{~ms}$, and 20 (7 male, 13 female) to $2000 \mathrm{~ms}$, respectively. All participants had normal or corrected to normal vision, and each received a monetary payment of NTD $\$ 100$ for their participation. It took about $30 \mathrm{~min}$ for participants to complete the experiment. As experiment 1, participants were recruited in accordance with

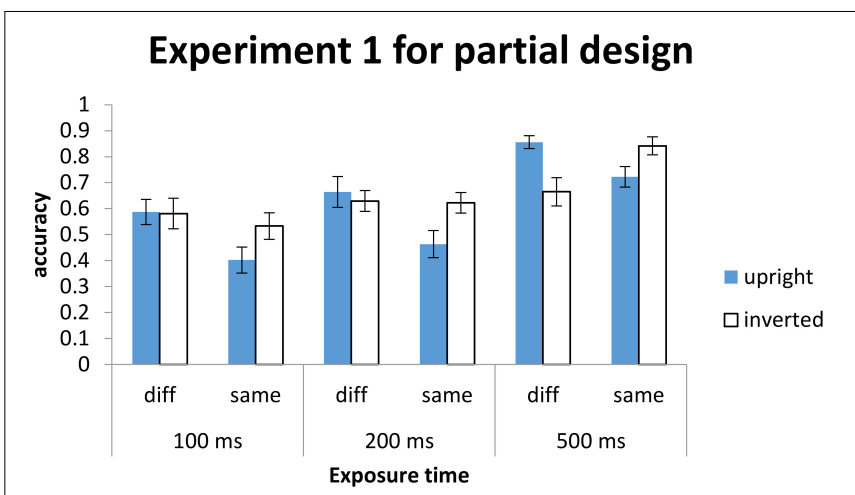

FIGURE 12 | The mean accuracy for response as a function of orientation for exposure time. Error bars indicate $\pm 1 \mathrm{SE}$, calculated within participants.

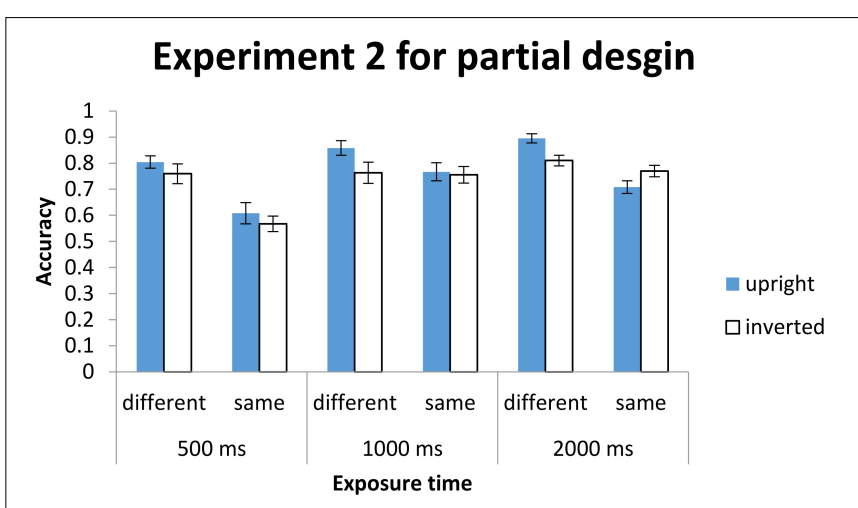

FIGURE 13 | The mean accuracy for response as a function of orientation for exposure time. Error bars indicate $\pm 1 \mathrm{SE}$, calculated within participants.

approval of the Research Ethics Committee of National Chung Cheng University, Chia-Yi, Taiwan (No. CCUREC104082101). Each participant had completed the informed consents when began the experiment.

\section{Stimuli and Apparatus}

The stimuli were the same as Experiment 1, except that each face image was made slightly bigger, which could be encircled by an (invisible) square with 100 pixels on each side. When presented on the display screen, each face had a width of about $5.1 \mathrm{~cm}$ and a height of about $6.1 \mathrm{~cm}$, extending a visual angle of about $4.01^{\circ} \times 4.8^{\circ}$ at a viewing distance of approximately $45 \mathrm{~cm}$.

A pair of faces were simultaneously presented on the display for a pre-determined duration of 500, 1,000, or 2,000 ms. Unlike Experiment 1 where face stimuli were presented at the same horizontal height, one face was located in the upper left quadrant and the other face was located in the lower right quadrant to discourage feature-by-feature comparisons. The center of the face located in left quadrant was about $4.69 \mathrm{~cm}$ below the top edge of the monitor, and about $13.31 \mathrm{~cm}$ to the right of left edge of the monitor. The center of the face located in the lower-right quadrant roughly had the same distances from the bottom and right edge of the monitor. The two faces were separated by a center-to-center distance of about $14.12 \mathrm{~cm}$. 


\section{Design and Procedure}

The same design used in Experiment 1 was used in Experiment 2, except for the exact length of exposure duration and the nature of manipulation. Specifically, three levels of relatively long exposure duration of 500, 1000, and $2000 \mathrm{~ms}$ was varied as a betweenparticipant factor. As in Experiment 1, the design of the present experiment is the complete design and computed differences in congruency effect between upright and inverted faces for each participant as the dependent measure for holistic processing.

\section{Procedure}

Each composite face was presented either upright or inverted for a total of 128 trials, with equal number of congruent and incongruent trials in accordance with the complete design. The upright face and inverted face conditions were separated into different blocks and the order of administration was counterbalanced across participants. In each trial, a "+" for fixation was first shown at the center of the display for $500 \mathrm{~ms}$, followed by the presentation of a pair of composite faces for one of the three durations, 500, 1000, or $2000 \mathrm{~ms}$, where exposure times was manipulated as a between-participants variable. Upon seeing the face pair, participants were asked to judge whether the top halves of the two faces were identical, while ignoring the bottom halves. The manipulation of face orientation was done as a within-participants variable by including two blocks of trials, one block with upright faces and the other with inverted faces. The order of administering the two blocks was counterbalanced across participants.

\section{Results}

The mean accuracy for correct judgment of the top halves of upright and inverted conditions, in terms of hit rate (HR) and correct rejection (CR) and $d^{\prime}$ derived from them, are shown in Table 2.

TABLE 2 | Descriptive statistics for each condition in Experiment 2.

\begin{tabular}{|c|c|c|c|c|c|}
\hline \multirow[t]{2}{*}{ Orientation } & \multirow[t]{2}{*}{ Congruent } & \multirow[t]{2}{*}{ Response } & \multicolumn{3}{|c|}{ Exposure duration } \\
\hline & & & $100 \mathrm{~ms}$ & $200 \mathrm{~ms}$ & $500 \mathrm{~ms}$ \\
\hline \multirow[t]{6}{*}{ Upright } & Congruent & $\mathrm{HR}$ & $0.68(0.14)$ & $0.86(0.12)$ & $0.89(0.13)$ \\
\hline & & $\mathrm{CR}$ & $0.80(0.11)$ & $0.86(0.12)$ & $0.90(0.16)$ \\
\hline & & $d^{\prime}$ & $1.51(0.75)$ & $2.59(0.89)$ & $3.03(1.14)$ \\
\hline & $\begin{array}{l}\text { Incongruent } \\
\text { trials }\end{array}$ & $\mathrm{HR}$ & $0.61(0.19)$ & $0.7(0.12)$ & $0.71(0.18)$ \\
\hline & & $\mathrm{CR}$ & $0.67(0.17)$ & $0.77(0.14)$ & $0.82(0.11)$ \\
\hline & & $d^{\prime}$ & $0.82(0.67)$ & $1.36(0.54)$ & $1.60(0.96)$ \\
\hline \multirow[t]{6}{*}{ Inverted } & Congruent trials & $\mathrm{HR}$ & $0.62(0.16)$ & $0.76(0.17)$ & $0.83(0.14)$ \\
\hline & & $\mathrm{CR}$ & $0.76(0.17)$ & $0.81(0.11)$ & $0.81(0.13)$ \\
\hline & & $d^{\prime}$ & $1.63(1.32)$ & $1.84(0.53)$ & $2.17(0.77)$ \\
\hline & $\begin{array}{l}\text { Incongruent } \\
\text { trials }\end{array}$ & $\mathrm{HR}$ & $0.57(0.14)$ & $0.71(0.15)$ & $0.77(0.15)$ \\
\hline & & $\mathrm{CR}$ & $0.74(0.14)$ & $0.76(0.13)$ & $0.83(0.09)$ \\
\hline & & $d^{\prime}$ & $0.87(0.46)$ & $1.36(0.54)$ & $1.85(0.65)$ \\
\hline
\end{tabular}

Means of hit rate (HR) and correct rejection (CR) for upright and inverted face condition in 500, 1000, and 2000 ms, respectively. Standard deviations are shown in parentheses.

\section{Holistic Processing}

A one-way ANOVA was conducted with exposure duration as the sole between-participants factor and its main effect of exposure time was significant, $F(2,55)=5.87, M S E=7.67, p<0.01$, $\eta_{\mathrm{p}}^{2}=0.176$. Post hoc comparisons with Bonferroni correction for multiple comparison $\left(\alpha_{B}=0.017\right)$ revealed that, as shown in Figure 10, the magnitude of holistic processing at $2000 \mathrm{~ms}$ was greater than that at $500 \mathrm{~ms}, p=0.001<\alpha_{B}=0.017$; no other comparisons were found significant $p$ s $>\alpha_{B}=0.017$, however.

In terms of planned comparisons, one-sample $t$-tests were performed, and used Holm-Bonferroni method to control the family wise error rate $\left(\alpha_{B}=0.017, \alpha_{B}=0.025, \alpha_{B}=0.05\right)$. The results showed holistic processing was not observed at the exposure duration of $500 \mathrm{~ms}, t<1$, but was found at both 1000 and $2000 \mathrm{~ms}\left\{1000 \mathrm{~ms}: t(16)=3.63, p<0.01<\alpha_{\mathrm{B}}=0.025\right.$, $M=0.75,95 \%$ CI $=[0.31,1.19] ; 2000 \mathrm{~ms}: t(19)=4.63$, $\left.p<0.001<\alpha_{\mathrm{B}}=0.017, M=1.12,95 \% \mathrm{CI}=[0.61,1.63]\right\}$.

\section{Part-Based Processing}

The same one-way ANOVA was conducted, with exposure duration as the between-participants factor, and as for holistic processing, its main effect was significant, $F(2,55)=7.42$, $M S E=3.14, p<0.01, \eta_{\mathrm{p}}^{2}=0.213$. Post hoc comparisons with Bonferroni correction for multiple comparison $\left(\alpha_{B}=0.017\right)$, as shown in Figure 11, revealed that performance at $500 \mathrm{~ms}$ was worse than those at $1000 \mathrm{~ms}\left(p=0.013<\alpha_{\mathrm{B}}=0.017\right)$ and $2000 \mathrm{~ms}$ $\left(p<0.01<\alpha_{\mathrm{B}}=0.017\right)$, and there was no significant difference between the latter two conditions $\left(p>\alpha_{B}=0.017\right)$.

In terms of planned comparisons, performances were better than chance at all three levels of exposure duration $\{500 \mathrm{~ms}$ : $t(20)=8.81, p<0.001<\alpha_{\mathrm{B}}=0.017, M=1.13,95 \% \mathrm{CI}=[0.86$, 1.39]; $1000 \mathrm{~ms}: t(16)=12.51, p<0.001<\alpha_{\mathrm{B}}=0.017$, $M=1.75,95 \% \mathrm{CI}=[1.46,2.05] ; 2000 \mathrm{~ms}: t(19)=10.85$, $\left.p<0.001<\alpha_{\mathrm{B}}=0.017, M=1.84,95 \% \mathrm{CI}=[1.49,2.20]\right\}$.

\section{Discussion}

In Experiment 2, holistic processing clearly is observed at $1000 \mathrm{~ms}$ and lasted till $2000 \mathrm{~ms}$ and perhaps even beyond. While this finding was consistent with the prediction made by Richler et al. (2009), it remains to be explained why the early study by Hole (1994) failed to find the composite face effect when participants saw two composite faces simultaneously with $2000 \mathrm{~ms}$ of exposure duration. There are three possibilities for the discrepancy: First, there was a clear difference regarding the nature of task and its design, where the present study was based on the complete design, but Hole (1994) adopted the traditional partial design.

Second, another possibility is the indices Hole chose were different from those chosen in the present study. Specifically, as measuring differences in congruency effect between upright and inverted condition, as proposed by Gauthier and Bukach (2007); in contrast, Hole (1994) used inversion superiority effect. Although Hole calculated reaction time to measure face inversion superiority, the results did not show reaction time (RT) because was not very useful to analyze reaction time data when the mean accuracy in a number of conditions were below 70\% (Tables 1, 2), 
which may well lead to biased estimate of RT performances. However, the results did find inversion superiority effect based on accuracy for exposure duration in $100 \mathrm{~ms}$ (see Figure 12 and Appendix 1), but failed to do so for exposure duration from 200 to $500 \mathrm{~ms}$ in Experiment 1 and 500 to $2000 \mathrm{~ms}$ in Experiment 2 (see Figure 13 and Appendix 2). In other words, it is relatively easy to detect inversion superiority effect with short exposure durations than with longer exposure durations.

Finally, according to Flin (1985) which suggested that stimuli discrimination may interfere with inversion effect. The mean accuracy for participants' performances was higher than $99 \%$ in the early study by Young et al. (1987), and it was similarly high in Hole's (1994) study (i.e., 88\%). These results suggest that the faces (and their composites) used in the earlier study were relatively easy for participants to judge, and if the composite faces were too hard to judge, inversion effect might not be obtained at all. As Flin had remarked, inversion effect cannot be detected if there were floor (or ceiling) effect, which points out the possibility that perceptual discriminability of face stimuli may modulate the presence as well as strength of holistic processing. However, there has been very little research to investigate systematically how perceptual discriminability may affect holistic processing.

\section{GENERAL DISCUSSION}

Taken together, the results from Experiments 1 and 2, revealed the separation between holistic processing and part-based processing, and as such gave credence to the dual-route model on face recognition envisioned by McKone (2010).

It is also interesting to note that at $500 \mathrm{~ms}$ of exposure duration, holistic processing was found in Experiment 1, but not in Experiment 2. There are some possibilities, for example, one possible explanation for the discrepancy is that holistic processing could be under the influence of spatial distance between a pair of stimulus faces. Another explanation is the design and the mask to cause the differences. In the future, it needs to be further manipulated for this issue.

In summary, the results of Experiments 1 and 2 indicated that holistic processing was maintained until $2000 \mathrm{~ms}$, supporting the prediction by Richler et al. (2009). However, it is not

\section{REFERENCES}

Amishav, R., and Kimchi, R. (2010). Perceptual integrality of componential and configural information in faces. Psychonom. Bull. Rev. 17, 743-748. doi: 10. 3758/PBR.17.5.743

Avidan, G., Tanzer, M., and Behrmann, M. (2011). Impaired holistic processing in congenital prosopagnosia. Neuropsychologia 49, 2541-2552. doi: 10.1016/j. neuropsychologia.2011.05.002

Bentin, S., Allison, T., Puce, A., Perez, A., and McCarthy, G. (1996). Electrophysiological studies of face perception in humans. J. Cogn. Neurosci. 8, 551-565. doi: 10.1162/jocn.1996.8.6.551

Bona, S., Cattaneo, Z., and Silvanto, J. (2016). Investigating the causal role of rOFA in holistic detection of Mooney faces and objects: an fMRI-guided TMS study. Brain Stimul. 9, 594-600. doi: 10.1016/j.brs.2016.04.003

Bruce, C., Desimone, R., and Gross, C. G. (1981). Visual properties of neurons in a polysensory area in superior temporal sulcus of the macaque. J. Neurophysiol. 46, 369-384. doi: 10.1152/jn.1981.46.2.369 supported that holistic processing would automatically occurred at part-base performance that was above chance. According to the third hypothesis (Figure 7) from Richler et al. (2009), they predicted that congruency effects are obtained as abovechance performance (of part-based processing) is observed and their results reveals that congruency effects were obtained in $50 \mathrm{~ms}$ to $800 \mathrm{~ms}$ when the performances were better than chance. In contrast, the results suggested that even when part-based performance was better than chance, holistic processing did not occur automatically. For example, with exposure durations of 1000 and $2000 \mathrm{~ms}$, holistic processing did occur. Rather, it seems that sufficient exposure duration is a key factor for producing evidence for holistic processing. In addition to exposure duration, if the spatial distance between two face stimuli was too great to see the pair of faces simultaneously, holistic processing may also be compromised. In the future, it is necessary to investigate systematically how perceptual discriminability and spatial factor may affect holistic processing.

\section{AUTHOR CONTRIBUTIONS}

C-CW contributed to the rationale of the whole experiments, analyzed the data, and drafted the manuscript.

\section{FUNDING}

This work was supported by Ministry of Science and Technology (MOST 108-2634-F-007-007) and the Yin Shu-Tien Educational Foundation.

\section{ACKNOWLEDGMENTS}

The author would like to express his sincere appreciation to his supervisor, Dr. Gary C.-W. Shyi, for his guidance, encouragement and continuous support. The author would also like to express his appreciation to the candid and constructive comments from two reviewers, which have greatly improved the manuscript.

Bruce, V., and Young, A. (1986). Understanding face recognition. Br. J. Psychol. 77, 305-327. doi: 10.1111/j.2044-8295.1986.tb02199.x

Bullier, J. (2001). Integrated model of visual processing. Brain Res. Rev. 36, 96-107. doi: 10.1016/S0165-0173(01)00085-6

Chua, K.-W., Richler, J. J., and Gauthier, I. (2014). Becoming a Lunari or Taiyo expert: learned attention to parts drives holistic processing of faces. J. Exp. Psychol. 40, 1174-1182. doi: 10.1037/a0035895

Desimone, R., Albright, T. D., Gross, C. G., and Bruce, C. (1984). Stimulusselective properties of inferior temporal neurons in the macaque. J. Neurosci. 4, 2051-2062. doi: 10.1523/JNEUROSCI.04-08-02051.1984

Diamond, R., and Carey, S. (1986). Why faces are not special: an effect of expertise. J. Exp. Psychol. 115, 107-117. doi: 10.1037/0096-3445.115.2.107

Farah, M. J., Wilson, K. D., Drain, M., and Tanaka, J. W. (1998). What is "special" about face perception? Psychol. Rev. 105, 482-498.

Fitousi, D. (2015). Composite faces are not processed holistically: evidence from the Garner and redundant target paradigms. Attent. Percept. Psychophys. 77, 2037-2060. doi: 10.3758/s13414-015-0887-4 
Fitousi, D. (2016). Comparing the role of selective and divided attention in the composite face effect: insights from Attention Operating Characteristic (AOC) plots and cross-contingency correlations. Cognition 148, 34-46. doi: 10.1016/j. cognition.2015.12.012

Flin, R. H. (1985). Development of face recognition: an encoding switch? Br. J. Psychol. 76, 123-134. doi: 10.1111/j.2044-8295.1985.tb01936.x

Galton, F. (1879). Composite portraits, made by combining those of many different persons into single resultant figure. J. Anthropol. Inst. 8, 132-144. doi: 10.2307/ 2841021

Gauthier, I., and Bukach, C. M. (2007). Should we reject the expertise hypothesis? Cognition 103, 322-330.

Gauthier, I., and Tarr, M. J. (2002). Unraveling mechanisms for expert object recognition: bridging brain activity and behavior. J. Exp. Psychol. 28, 431-446. doi: 10.1037/0096-1523.28.2.431

Gauthier, I., Tarr, M. J., and Bubb, D. (2010). Perceptual Expertise: Bridging Brain and Behavior. Oxford: Oxford University Press.

Green, D. M., and Swets, J. A. (1966). Signal Detection Theory and Psychophysics. New York, NY: Wiley.

Hadad, B.-S., and Kimchi, R. (2006). Developmental trends in utilizing closure for grouping of shape: effects of spatial proximity and collinearity. Percept. Psychophys. 68, 1264-1273. doi: 10.3758/BF03193726

Hole, G. J. (1994). Configurational factors in the perception of unfamiliar faces. Perception 23, 65-74. doi: 10.1068/p230065

Jacques, C., and Rossion, B. (2006). The speed of individual face categorization. Psychol. Sci. 17, 485-492. doi: 10.1111/j.1467-9280.2006.01733.x

Johnson, M. H., and Morton, J. (1991). Biology and Cognitive Development: The Case of Face Recognition. Oxford: Blackwell.

Jonas, J., Descoins, M., Koessler, L., Colnat-Coulbois, S., Sauvée, M., Guye, M., et al. (2012). Focal electrical intracerebral stimulation of a face-sensitive area causes transient prosopagnosia. Neuroscience 11, 281-288. doi: 10.1016/ j.neuroscience.2012.07.021

Jonas, J., Rossion, B., Krieg, J., Koessler, L., Colnat-Coulbois, S., Vespignani, H., et al. (2014). Intracerebral electrical stimulation of an occipital face-selective area impairs individual face discrimination. Neuroimage 99, 487-497. doi: 10. 1016/j.neuroimage.2014.06.017

Kanwisher, N., McDermott, J., and Chun, M. M. (1997). The fusiform face area: a module in human extrastriate cortex specialized for face perception. J. Neurosci. 17, 4302-4311. doi: 10.1523/JNEUROSCI.17-11-04302.1997

Kobatake, E., and Tanaka, K. (1994). Neuronal selectivities to complex object features in the ventral visual pathway of the macaque cerebral cortex. J. Neurophysiol. 71, 856-867. doi: 10.1152/jn.1994.71.3.856

Maurer, D., Grand, R. L., and Mondloch, C. J. (2002). The many faces of configural processing. Trends Cogn. Sci. 6, 255-260. doi: 10.1016/S1364-6613(02)01903-4

McKone, E. (2010). "Face and object recognition: how do they differ?" in Tutorials in Visual Cognition ed. V. Coltheart. London: Psychology Press, 261-303.

McKone, E., Davies, A. A., Darke, H., Crookes, K., Wickramariyaratne, T., Zappia, S., et al. (2013). Importance of the inverted control in measuring holistic face processing with the composite effect and part-whole effect. Front. Psychol. 4:33. doi: 10.3389/fpsyg.2013.00033

McKone, E., and Yovel, G. (2009). Why does picture-plane inversion sometimes dissociate perception of features and spacing in faces, and sometimes not? Toward a new theory of holistic processing. Psychonom. Bull. Rev. 16, 778-797. doi: 10.3758/PBR.16.5.778

McNeil, J. E., and Warrington, E. K. (1993). Prosopagnosia: a face-specific disorder. Q. J. Exp. Psychol. 46, 1-10. doi: 10.1080/14640749308401064

Mondloch, C. J., Le Grand, R., and Maurer, D. (2002). Configural face processing develops more slowly than featural face processing. Perception 31, 553-566. doi: $10.1068 / \mathrm{p} 3339$

Moscovitch, M., Winocur, G., and Behrmann, M. (1997). What is special about face recognition? Nineteen experiments on a person with visual object agnosia and dyslexia but normal face recognition. J. Cogn. Neurosci. 9, 555-604. doi: 10.1162/jocn.1997.9.5.555
Richler, J. J., and Gauthier, I. (2014). A meta-analysis and review of holistic processing. Psychol. Bull. 140, 1281-1302. doi: 10.1037/a0037004

Richler, J. J., Mack, M. L., Gauthier, I., and Palmeri, T. J. (2009). Holistic processing of faces happens at a glance. Vision Res. 49, 2856-2861. doi: 10.1016/j.visres. 2009.08.025

Richler, J. J., Mack, M. L., Palmeri, T. J., and Gauthier, I. (2011). Inverted faces are (eventually) processed holistically. Vision Res. 51, 333-342. doi: 10.1016/j.visres. 2010.11.014

Richler, J. J., Palmeri, T. J., and Gauthier, I. (2012). Meanings, mechanisms, and measures of holistic processing. Front. Psychol. 3:553. doi: 10.3389/fpsyg.2012. 00553

Robbins, R., and McKone, E. (2007). No face-like processing for objects-ofexpertise in three behavioural tasks. Cognition 103, 34-79. doi: 10.1016/j. cognition.2006.02.008

Rossion, B. (2008). Picture-plane inversion leads to qualitative changes of face perception. Acta Psychol. 128, 274-289. doi: 10.1016/j.actpsy.2008.02.003

Rossion, B. (2009). Distinguishing the cause and consequence of face inversion: the perceptual field hypothesis. Acta Psychol. 132, 300-312. doi: 10.1016/j.actpsy. 2009.08.002

Rossion, B. (2013). The composite face illusion: a whole window into our understanding of holistic face perception. Vis. Cogn. 21, 139-253. doi: 10.1080/ 13506285.2013.772929

Rossion, B., and Boremanse, A. (2008). Nonlinear relationship between holistic processing of individual faces and picture-plane rotation: evidence from the face composite illusion. J. Vision 8, 1-13. doi: 10.1167/8.4.3

Tanaka, J. W., and Farah, M. J. (1993). Parts and wholes in face recognition. Q. J. Exp. Psychol. 46, 225-245. doi: 10.1080/14640749308401045

Tanaka, J. W., and Gordon, I. (2011). "Feature, configuration, and holistic processing," in The Oxford Handbook of Face Perception, eds A. J. Calder, G. Rhodes, M. Johnson, and J. Haxby (New York, NY: Oxford University Press), 177-179.

Tanaka, J. W., Kay, J. B., Grinnell, E., Stansfield, B., and Szechter, L. (1998). Face recognition in young children: when the whole is greater than the sum of its parts. Vis. Cogn. 5, 479-496. doi: 10.1080/713756795

Tsao, D. Y., Freiwald, W. A., Tootell, R. B., and Livingstone, M. S. (2006). A cortical region consisting entirely of face selective cells. Science 311, 670-674. doi: 10.1126/science.1119983

Tsao, D. Y., and Livingstone, M. S. (2008). Mechanisms for face perception. Annu. Rev. Neurosci. 31, 411-438. doi: 10.1146/annurev.neuro.30.051606.09 4238

Wenger, M. J., and Ingvalson, E. M. (2003). Preserving informational separability and violating decisional separability in facial perception and recognition. J. Exp. Psychol. 29, 1106-1118. doi: 10.1037/0278-7393.29.6.1106

Yin, R. K. (1969). Looking at upside-down faces. J. Exp. Psychol. 81, 141-145. doi: $10.1037 / \mathrm{h} 0027474$

Young, A. W., Hellawell, D., and Hay, D. C. (1987). Configurational information in face perception. Perception 16, 747-759. doi: 10.1068/p160747

Zhao, M., Bülthoff, H. H., and Bülthoff, I. (2015). Beyond faces and expertise: facelike holistic processing of nonface objects in the absence of expertise. Psychol. Sci. 27, 213-222. doi: 10.1177/0956797615617779

Conflict of Interest Statement: The author declares that the research was conducted in the absence of any commercial or financial relationships that could be construed as a potential conflict of interest.

The reviewer YU and handling Editor declared their shared affiliation.

Copyright (c) 2019 Wang. This is an open-access article distributed under the terms of the Creative Commons Attribution License (CC BY). The use, distribution or reproduction in other forums is permitted, provided the original author(s) and the copyright owner(s) are credited and that the original publication in this journal is cited, in accordance with accepted academic practice. No use, distribution or reproduction is permitted which does not comply with these terms. 


\section{APPENDIX}

\section{Appendix 1: Experiment 1}

There was a $2 \times 2 \times 3$ repeated-measured ANOVAs conducted with Orientation (upright/inverted), Exposure time (100, 200, and $500 \mathrm{~ms}$ ) and Response (same/different) as within-participant variables. A significant main effect of exposure time was found, $F(2,34)=92.2, M S E=1.15, p<0.001, \eta_{\mathrm{p}}^{2}=0.844$, which was modulated by two-way interaction with orientation, $F(2,34)=5.14, M S E=0.058, p<0.05, \eta_{\mathrm{p}}^{2}=0.232$. A simple main effect revealed that the performance of inverted faces $(M=0.56)$ was better than that of upright faces $(M=0.49)$ in $100 \mathrm{~ms}$ $(p<0.01)$, but there were no significant differences in 200 and $500 \mathrm{~ms}(p s>0.05)$. As a result, inversion superiority effect was found in $100 \mathrm{~ms}$. There was a significant two-way interaction between orientation and response, $F(1,17)=23.23, M S E=0.61$, $p<0.001, \eta_{\mathrm{p}}^{2}=0.577$. The difference between accuracy of different and same trials in the upright faces $(M=0.17)$ was greater than that in the inverted faces $(M=-0.04)$. No other main effects and interactions were found.

\section{Appendix 2: Experiment 2}

The mean accuracy in each condition was first submitted to a three-way mixed-measured analysis of variance (ANOVA) with face orientation (upright vs. inverted) and response (same vs. different) as within-participant variables and exposure duration (500, 1000, and $2000 \mathrm{~ms})$ as between-participants variable. The results showed that performance on upright trials $(M=0.77)$ was better than that on inverted trials $(M=0.74)$, as reflected in a significant main effect of orientation, $F(1,55)=9.19$, $M S E=0.07, p<0.001, \eta_{\mathrm{p}}^{2}=0.143$. However, inversion superiority effect was not found. There was also a main effect of response, $F(1,55)=26.07, M S E=0.82, p<0.001, \eta_{p}^{2}=0.322$. A main effect of exposure time was found $[F(1,55)=9.92$, $\left.M S E=0.31, p<0.001, \eta_{\mathrm{p}}^{2}=0.265\right]$. Although two-way interaction between orientation and response was found, $F(1,55)=4.15$, $M S E=0.89, p<0.05, \eta_{\mathrm{p}}^{2}=0.07$, the difference between accuracy of different trials and same trials in the upright faces $(M=0.16)$ was greater than that of the inverted face $(M=09)$. The present study also reveals the two-way interaction between exposure time and response $[F(2,55)=3.19, M S E=0.10$, $p<0.05, \eta_{\mathrm{p}}^{2}=0.104$ ], but two-way interaction between orientation and exposure time was not found. No three-way interaction was found $[F(1,55)=1.22, M S E=0.026, p>0.05]$. According to these results, inversion superiority effect cannot be obtained. 